\title{
A new sensorless speed control structure for PMSM using reference model
}

\author{
K. URBANSKI \\ Institute of Control and Information Engineering, Poznan University of Technology, 3A Piotrowo St., 61-138 Poznan, Poland
}

\begin{abstract}
In the paper a new sensorless control structure for the PMSM drive is presented. Such a structure is especially recommended for speed in the range of single revolutions per second (excluding standstill). The method uses a back EMF observer for position estimation. However, there is no need to estimate the speed. This is a big advantage because of possible irregularity of estimated back EMF in this speed range, which makes the calculation of speed difficult or impossible. The proposed structure is similar to the model following control, but the reference model attempts to track the motor operating point. The main idea is to utilize as a reference a model of the whole drive, including speed and current controllers and motor model. Such a model produces reference voltage for the real inverter. However, an extra unit - called rotator - is needed to provide precise control of direct axis current, which is sensitive to the improper position of the voltage vector. The rotator acts as a kind of compensator for error of position estimation.
\end{abstract}

Key words: permanent magnet motors, sensorless control, observers, variable speed drives.

\section{Introduction}

The high power density, high torque to inertia ratio and small torque ripple are the advantages of the permanent magnet synchronous motor (PMSM). To achieve good dynamics, especially during transients, in the case of rapid reference speed changes and load changes, a vector control should be used. Shaft position information is required to enable the effective vector control of a PMSM drive. In a typical realization of the control structure, the sensorless operation of a speed control for a PMSM drive may be achieved simply by eliminating the position sensor and by the use of a position estimator instead. This approach works well for medium and high speed ranges [1-6], even in the case of more complex drives, e.g., for a motor and inverter connection via motor choke $[7,8]$ or sinusoidal filter $[9,10]$. Another situation is in the case of a low speed range [11]. In such a case of operation, in many applications there is a need to introduce a special control algorithm $[12,13]$, as well as the use of a sensorless control. It is assumed that position estimation based on the back electromotive force (EMF) does not have sufficient accuracy at lower speed range $[14,15]$. In such case other methods are used, e.g., using injection of additional test signals $[16,17]$. For speed in the range several revolutions per second, the calculation of speed directly from estimated position value is not so trivial because of the possibility of excitation oscillations caused by the speed estimator. This happens due to the non-sinusoidal shape of the estimated back EMF, which has a major impact on the stable sensorless performance of the drive at this speed range. However, the position calculation based on the back EMF estimation may work correctly even in such speed

*e-mail: konrad.urbanski@put.poznan.pl

Manuscript submitted 2016-07-08, revised 2016-10-12, 2016-11-20 and 2017-01-06, initially accepted for publication 2017-01-30, published in August 2017. range. This is confirmed by experimentally obtained waveforms shown in Fig. 1 and Fig. 2. This is the idle speed, using a constant value of reference current on $q$ axis. These waveforms were obtained for a traditional field oriented control (FOC) method of the PMSM (using a similar structure as is shown in Fig. 4), with a cascade control structure using a closed speed control loop (which was disconnected for the test) with inner current control loops containing separate controllers on $d$ and $q$ axes, rotating synchronously with the rotor. The overall structure of control utilizes the sine and cosine of the estimated position and calculated speed value instead of measured values. To obtain smooth output values from the observer, the reference voltage is used instead of measured ones. This simplification gives good results
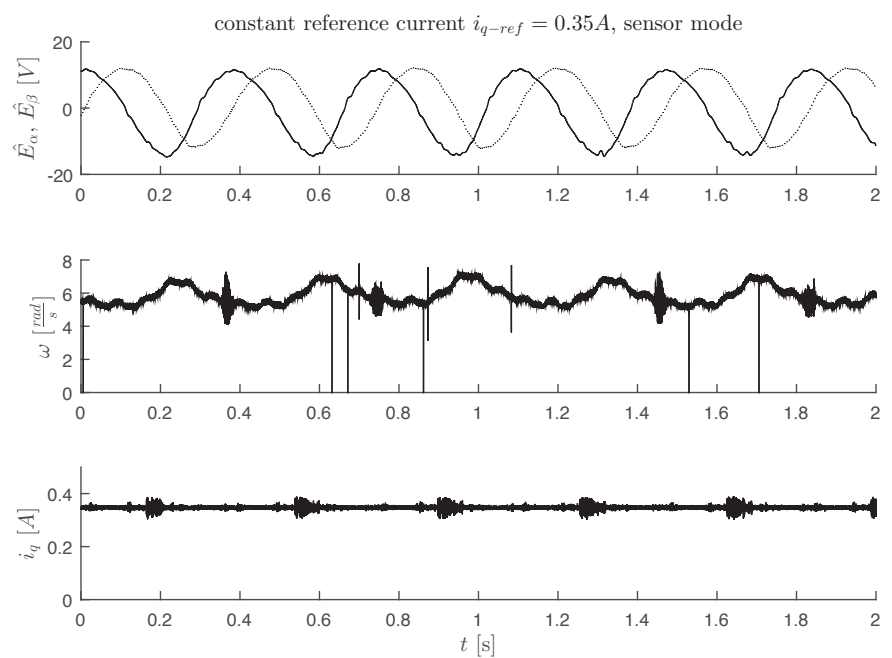

Fig. 1. Typical control structure, sensor mode: constant reference current $i_{q}=0.35 \mathrm{~A}$, up: estimated back EMF $-\hat{E}_{\alpha}, \hat{E}_{\beta}$, middle: measured speed (average value $6 \frac{\mathrm{rad}}{\mathrm{s}}$, down: measured current $i_{q}$ 

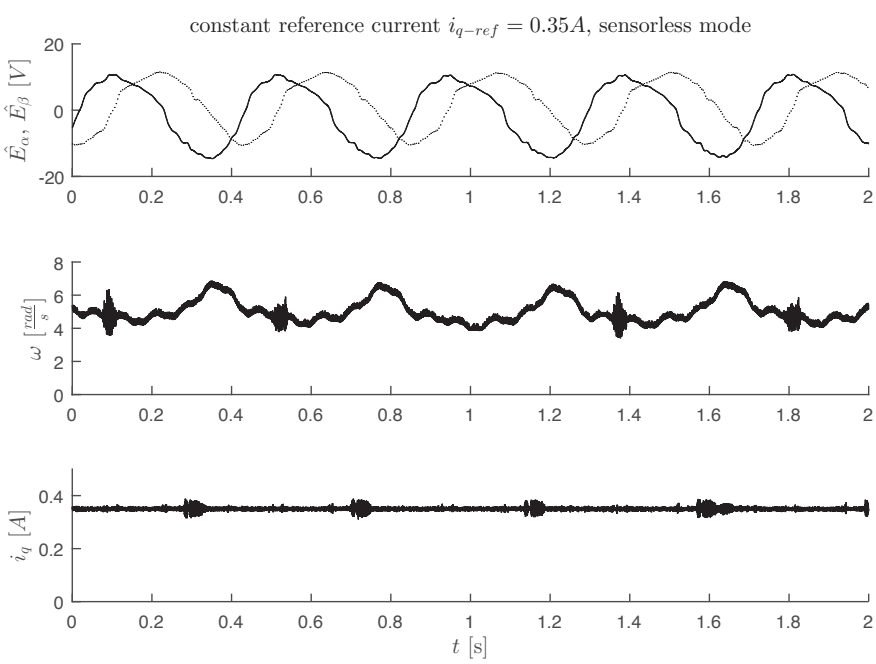

Fig. 2. Typical control structure, sensorless mode: constant reference current $i_{q}=0.35 A$, up: estimated back EMF $-\hat{E}_{\alpha}, \hat{E}_{\beta}$, middle: measured speed (average value $5 \frac{\mathrm{rad}}{\mathrm{s}}$ ), down: measured current $i_{q}$

in the medium and high speed range. However, in the low speed range, where requested voltage is low, the reference voltage may differ noticeably from the inverter output voltage, which affects the estimated back EMF amplitude. The sensor and sensorless operation, where a constant value of current on $q$ axis is the setpoint, produces almost equally shaped speed waveforms. It is noticeable, that the problem lies not in the position estimation, but there is essentially a fast and stable speed calculation. The nonlinearity of the motor is extremely influential in this speed range. This is indicated by the shape of the speed waveforms, obtained in the case of constant current value. Such a phenomenon particularly affects a drive with low moment of inertia. For such a drive, in typical realization, the speed should be estimated with a much smaller delay than the period of a speed fluctuation (which is shown in Fig. 2) to provide an adequate response of the speed controller without the excitation of the speed oscillation. An adequate reference current in $q$ axis may be processed properly by P/PI controller only with fast settings, which makes it practically impossible to work in the sensorless mode on a drive with low moment of inertia (sensorless control usually requires slowing down of the speed controller). Therefore, a sensorless control structure for speed has been developed which avoids the need to calculate speed from the estimated back EMFs. This is the main difference to the standard method, and it is a big advantage, because it allows the avoidance of problems in the case of speed estimation in this speed range. The proposed control structure uses a "virtual" speed, prepared in the reference drive model (instead of estimated speed), and a position observer is used to improve the accuracy of positioning the appropriate vectors in the (real) motor.

\section{Reference based control structures}

There are many structures known in the area of motion control, which use a reference value source. Some are used typically in sensor mode to improve performance and some may be used as an estimator. The most similar structure to the one presented in the paper - principally by the basic idea - is the MFC (Model Following Control) [18] and its modifications, as an adaptive MFC (AMFC) [19]. The main comparison of the proposed control system and the MFC is described in the next section. The other structures, MRAS (Model Reference Adaptive System) [20] and MRAC (Model Reference Adaptive Control) [21], and the IMC (Internal Model Control) [22, 23] are used mainly in order to estimate the unknown component of the state vector.

\section{The new control structure}

In short, in a typical MFC structure, the main controller generates a control signal for the object and its model. The additional controller, fed by a difference signal between the object output and model output, generates an additional control signal, which is added into object input. Hence, if a tracking error occurs, an additional control signal is generated, whose task is to decrease the tracking error rapidly - and the object must follow the model.

In the presented control structure, the object (PMSM) follows the reference model. However, if a tracking error occurs, a reference model tries to fit to the object operating point, due to the influence from the estimated load torque and the velocity correction signals in the reference model. For example, in the case of a step change in the motor load, the motor begins to slow down, and the motor model in the reference begins to slow down also, because of the load estimator, which influences the drive model. Thus, the impact of distortion is eliminated by internal controllers in the drive model. The control structure consists of the following elements:

- adder for correcting the reference speed (Fig. 3: unit 1)

- reference model of the drive (Fig. 3: unit 2)

- factor $k$ affects the dynamics of the rotator operation (Fig. 3: unit 3)

- rotator (Fig. 3: unit 4)

- transformation block $\alpha \beta \rightarrow a b c$ (Fig. 3: unit 5)

- inverter (Fig. 3: unit 6)

- transformer (Fig. 3: unit 7)

- currents measurement (Fig. 3: unit 8)

- PMSM (Fig. 3: unit 9)

- transformation block $a b c \rightarrow \alpha \beta$ (Fig. 3: unit 10)

- transformation block $\alpha \beta \rightarrow d q$ (Fig. 3: unit 11)

- position observer (Fig. 3: unit 12)

- current $i_{d}$ corrector (Fig. 3: unit 13)

- adder for correcting the angle of reference voltage vector (Fig. 3: unit 14)

- load estimator (Fig. 3: unit 15)

- adder for the load estimator (Fig. 3: unit 16).

Advantages of this sensorless structure are particularly visible in the low speed range. The system operates in the following manner: the reference model of the drive generates the reference voltage $u_{\alpha-r e f}, u_{\beta-r e f}$ and the reference currents $i_{d-r e f}$ and $i_{q-r e f}$, based on the corrected speed reference signal $\omega_{\text {ref } 2}$ and the estimated value of load torque $\hat{T}_{L}$. Reference voltages 


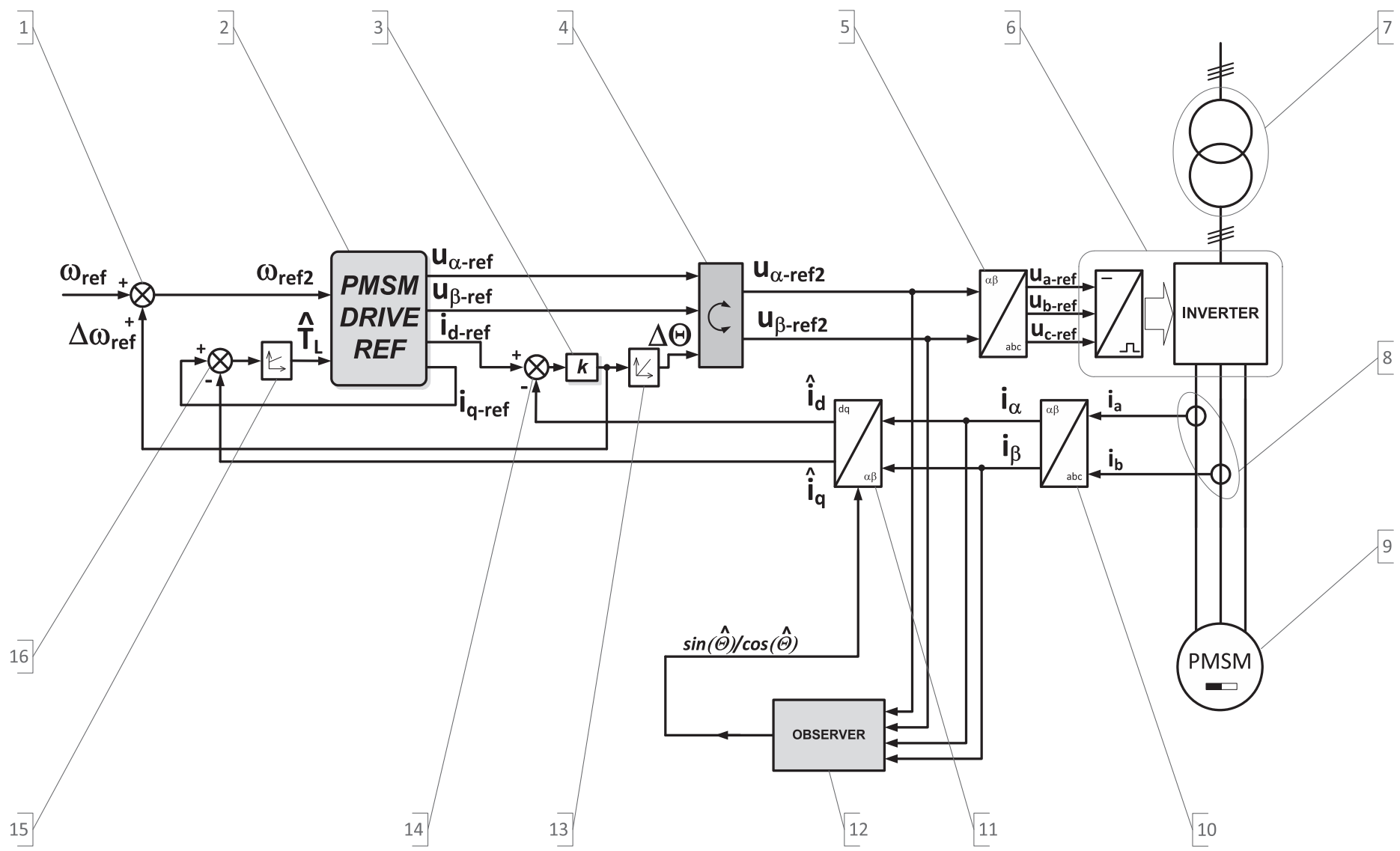

Fig. 3. A general view of the control structure

are used to prepare the supply voltage vector and the reference currents (which means the "measured" currents in the drive model) are used to increase the accuracy of the control. The position observer estimates the sine and cosine of the shaft position, which is used in a coordinate system transformation block.

One of the most important units is the rotator. It rotates the reference voltage vector by angle $\Delta \Theta$, which is generated by the current $i_{d}$ corrector. The input voltage vector and the output voltage vector may rotate at a different angular speed. This is a normal phenomena due to the limited accuracy of the model and the limited accuracy of the position estimation. The difference in the speed is the result of variation in time of the $\Delta \Theta$ value. The current $i_{d}$ corrector produces the $\Delta \Theta$ angle used in the rotator. It compares the current $i_{d-r e f}$, which is generated by the reference model, and the current $\hat{i}_{d}$, which is calculated on the basis of measured currents and estimated position. If a difference occurs, the corrector changes the $\Delta \Theta$ angle to decrease the error.

The next important unit, the load estimator, helps the reference model of the drive to fit to the (real) motor operating point in the case of a load torque change. Its operation is based on comparing the torque currents $i_{q}$ from the reference model and the calculated value based on measured currents, which are converted using a position estimated in the observer. In addition, a correcting signal of speed $\Delta \omega_{\text {ref }}$ is introduced in the summing junction of the referenced speed (Fig. 3: unit 1), which increases speed control accuracy in the case of the presence of a load torque. It is important to notice that this is not the estimated speed, but the correction value. The practical advantage of this control structure is that there is no need to estimate the speed. However, it is still the speed vector control structure. This means, that it gives the possibility to control the speed quickly and adequately, even in cases of rapid changes of load or reference speed.

3.1. Reference model. As a reference model of a drive with PMSM, as simple as the possible model may be used. However, the complexity of the model affects the ability to accurately track the model by the real system. The model used in the proposed control system is a typical vector control structure of a PMSM drive. The only difference is the lack of an inverter model, in order to simplify the drive model. The presented control system uses the traditional field oriented control method for the PMSM (Fig. 4) as a reference source, with cascade control structure, using a closed speed control loop $\left(R \_w-\right.$ speed controller) with inner current control loops containing separate controllers on $d$ axis ( $R \_i d$ block) and $q$ axis ( $R \_i q$ block) rotating synchronously with the rotor. All controllers have a PI structure with limited output. The following names of inputs and outputs of Fig. 4, correspond to the names of inputs and outputs of unit 2 in Fig. 3: the "wref2" input (Fig. 4) acts as $\omega_{\text {ref } 2}$ input (Fig. 3), the "TL" ${ }^{\wedge}$ input (Fig. 4) acts as $\widehat{T}_{L}$ (estimated 


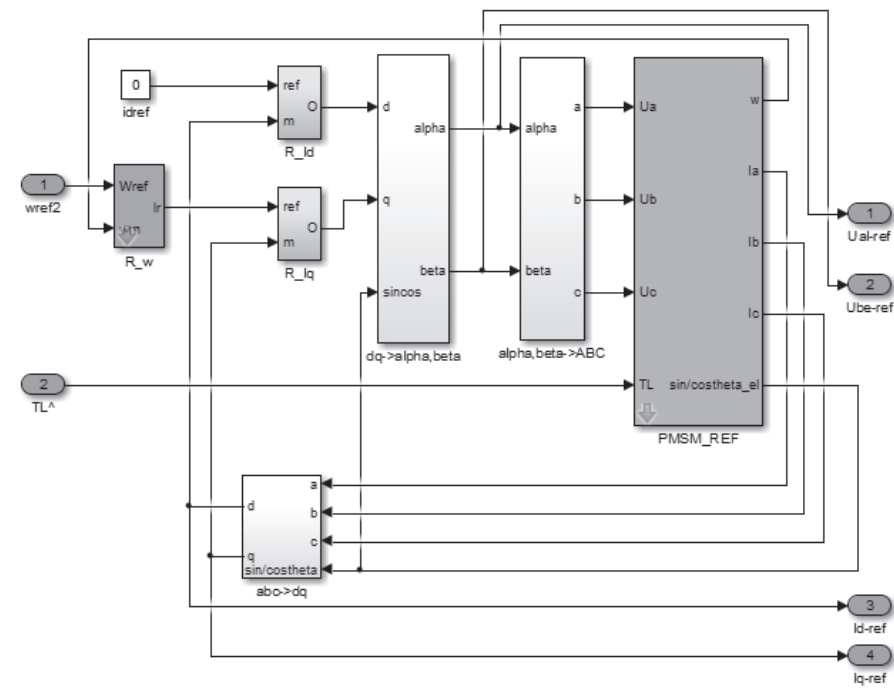

Fig. 4. A general view of the control structure of the reference model

load torque) input (Fig. 3). The outputs $u_{\alpha-r e f}, u_{\beta-r e f}, i_{d-r e f}, i_{q-r e f}$ (Fig. 3 unit 2) are converted inputs and outputs of the PMSM model "PMSM_REF" (Fig. 4). The inverter's transfer function is simplified to unity. That system is realized as a sensor based system. The PMSM model was prepared using ordinary simplified assumptions: there are no windings in the rotor, eddy currents and the effect of temperature are neglected, the flux produced by the rotor is constant [24]. The general form of the PMSM model in a system with $d q$ coordinates rotating with the rotor can be expressed as follows:

$$
\begin{gathered}
u_{d}=R_{s} \cdot i_{d}+L_{d} \cdot \frac{d i_{d}}{d t}-p \cdot \Psi_{q} \cdot \omega, \\
u_{q}=R_{s} \cdot i_{q}+L_{q} \cdot \frac{d i_{q}}{d t}+p \cdot \Psi_{d} \cdot \omega, \\
\Psi_{d}=L_{d} \cdot i_{d}+\Psi_{f}, \\
\Psi_{q}=L_{q} \cdot i_{q}, \\
T_{e}=p \cdot \frac{3}{2}\left[\Psi_{d} \cdot i_{q}-\Psi_{q} \cdot i_{d}\right], \\
J \cdot \frac{d \omega}{d t}=T_{e}-T_{L},
\end{gathered}
$$

where $u_{d}, u_{q}$ means the components of input voltage; $i_{d}, i_{q}$ means the components of current; $L_{d}, L_{q}$ means inductance of the motor on the $d q$ axis respectively (in this case $L_{d}=L_{q}$ ); $\Psi_{d}, \Psi_{q}, \Psi_{f}$, means flux on the $d q$ axis respectively and flux excited by permanent magnet. The electromagnetic torque is represented by the symbol $T_{e}$ and load torque by the symbol $T_{L}$; $\omega$ is the mechanical speed, and $p$ is the number of pole pairs. Symbol $R_{S}$ means the windings resistance, and $J$ is the total moment of inertia. Voltages $u_{d}, u_{q}$ are used after transformation into the stationary coordinate system as the $u_{\alpha-r e f}$ and $u_{\beta-r e f}$ outputs. Currents $i_{d}, i_{q}$ are used as the reference values $i_{d-r e f}$ and $i_{q-r e f}$.

The reference model was prepared using Matlab-Simulink environment. Then the $\mathrm{C}$-code was generated, using tools provided by Matlab. The code obtained was implemented in the microprocessor of the laboratory stand as a part of the control program.

3.2. Position observer. In order to estimate the shaft position, in the presented control structure a Luenberger observer is used [25]. However, the type of observer is not important in the context of the control system as a whole. There is a possibility to use an observer which estimates the position using any method: using signal injections or the observers, e.g., based on back EMF estimation, using Luenberger and modified Luenberger observers [26], based on various realizations of Kalman filter [27-30], using sliding mode observers [31-33] and artificial neural networks [34]. As mentioned above, the control system utilizes the Luenberger observer. The observer estimates the back EMFs in a stator based coordinate system $\alpha \beta$ and calculates sine and cosine of the rotor shaft position. The proportional form of the correction function of the observer is enough in the case of the low speed range. In a typical realization, assuming that the derivative of disturbances is equal to zero, one can write the observer equations as:

$$
\begin{gathered}
\frac{d \hat{i}_{\alpha}}{d t}=-\frac{R_{s}}{L_{s}} \cdot \hat{i}_{\alpha}-\frac{1}{L_{s}} \cdot \hat{e}_{\alpha}+\frac{1}{L_{s}} \cdot u_{\alpha}+K_{i \alpha}\left(\hat{i}_{\alpha}-i_{\alpha}\right), \\
\frac{d \hat{i}_{\beta}}{d t}=-\frac{R_{s}}{L_{s}} \cdot \hat{i}_{\beta}-\frac{1}{L_{s}} \cdot \hat{e}_{\beta}+\frac{1}{L_{s}} \cdot u_{\beta}+K_{i \beta}\left(\hat{i}_{\beta}-i_{\beta}\right), \\
\frac{d \hat{e}_{\alpha}}{d t}=K_{e \alpha}\left(\hat{i}_{\alpha}-i_{\alpha}\right), \\
\frac{d \hat{e}_{\beta}}{d t}=K_{e \beta}\left(\hat{i}_{\beta}-i_{\beta}\right),
\end{gathered}
$$

where $u_{\alpha}, u_{\beta}$ mean the components of input voltage (in the presented system, a reference voltage obtained from the current controller outputs is used instead); $\hat{i}_{\alpha}, \hat{i}_{\beta}, i_{\alpha}, i_{\beta}$ are the components of (estimated and measured) currents; $\hat{e}_{\alpha}$ and $\hat{e}_{\beta}$ mean estimated back EMF in the $\alpha \beta$ axis respectively. The position is calculated from estimated values of the back EMFs using simplified relationship $(11,12)$ :

$$
\begin{aligned}
& \sin (\hat{\Theta})=-\frac{\hat{e}_{\alpha}}{|\hat{e}|} \\
& \cos (\hat{\Theta})=\frac{\hat{e}_{\beta}}{|\hat{e}|}
\end{aligned}
$$

3.3. Rotator and current $i_{d}$ corrector. A new type of control path used in the speed control system is the rotator group. It consists of a block named "rotator" and its controller. This controller, or rather the corrective device, modifies the reference voltage vector angle in regard to the notified current error in $d$ axis: $i_{d-r e f}-\hat{i}_{d}$ (Fig. 3). A rotator turns the reference voltage vector 
$\left\{u_{\alpha-r e f}, u_{\beta-r e f}\right\}$ into the exact position $\left\{u_{\alpha-r e f 2}, u_{\beta-r e f 2}\right\}$, relative to the shaft position. As an accurate position in a constant torque region the position is assumed for which the $d$ axis current error is the smallest. The new reference voltage vector, whose position is corrected by angle $\Delta \Theta$ (generated by the current $i_{d}$ corrector), is calculated using formulas:

$$
\begin{aligned}
& u_{\alpha-r e f 2}=u_{\alpha-r e f} \cdot \cos (\Delta \Theta)-u_{\beta-r e f} \cdot \sin (\Delta \Theta) \\
& u_{\beta-r e f 2}=u_{\alpha-r e f} \cdot \sin (\Delta \Theta)-u_{\beta-r e f} \cdot \cos (\Delta \Theta)
\end{aligned}
$$

The current $i_{d}$ corrector is an integral type controller, with a special kind of limit - where its output is limited to the range $0 \div 2 \pi$, using modulo of $2 \pi$. This controller output signal may change the value by twisting its value at boundaries $0 \Leftrightarrow 2 \pi$. In the ideal case, at a steady state, after finding the proper $\Delta \Theta$, this value stops changing. In a real case, at a steady state, there is always a small difference $i_{d-r e f}-\hat{i}_{d}$, which is still integrated. The effect shows that the input voltage vector and the output voltage vector may rotate with different angular speeds. The sign and value of this speed difference is influenced by the reference model accuracy and the position estimation accuracy. In the case of weak position estimation, an additional adjustment for $i_{d}$ corrector is necessary. The simplest method of such adjustment is to link the correction value with the current on $q$ axis by constant factor $k 2$ (Fig. 5). However, in the wider range of operation, especially in the case of weak position estimation accuracy, or in the case of estimation of motor parameters with low accuracy, a gain $k 2$ should be converted into a function of the operating point, e.g., $k 2=f\left(\omega_{\text {ref }}, T_{L}\right)$.

3.4. Load estimator. Looking at the structure of a MFC, one can see that the process (PMSM) interacts with the main controller (the reference source) by the correcting reference speed (additional speed feedback from the unit 3, Fig. 3) and by the estimated load torque, which affects the drive model. These two signals force a change of the operating point of the reference model due to load torque appearance. The load torque estimator is implemented as a PI structure. Its input signal is calculated as the difference between the referenced and estimated current: $i_{q-r e f}-\hat{i}_{q}$. If this difference occurs, it means that there is a difference in torque values between the model and the real motor.

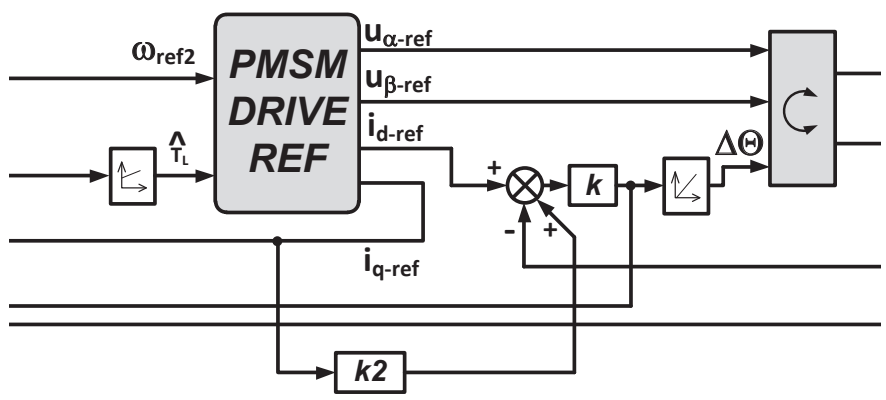

Fig. 5. Part of control structure with modification for compensation of position error estimation. The rest of the control structure is similar to that in Fig. 3
Therefore, the torque of the motor model is modified so that the operating point of the model comes close to the operating point of the real motor. Presently, the parameters of the load estimator are determined experimentally. This is safe to implement because if the load estimator is too slow, it does not cause instability, but only affects the steady state speed error in the case of a loaded motor (Fig. 8).

\section{Simulations}

This control structure has the possibility to work properly with or without the load estimator, and with or without the reference speed correction (the combination of "activated correction speed" and "deactivated load torque estimation" is not recommended without additional correction of parameters for units 3 and 13). However, the lack of these correction loops affects the drive dynamics or can cause the presence of steady state speed error under load, as is presented in Figs. 6-8. The simulations were made using Matlab Simulink environment. The motor model (as a reference and "real" motor also) and observer

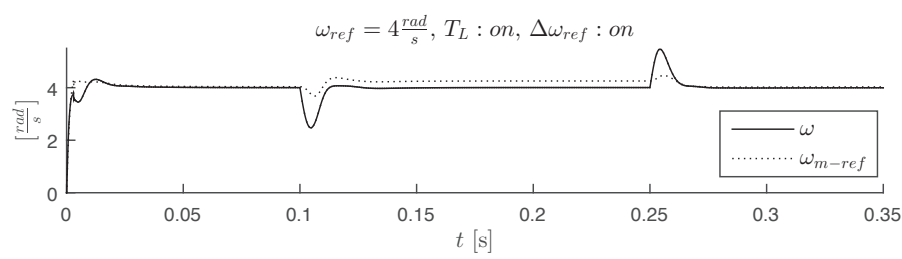

Fig. 6. Simulation, measured speed $\omega$ and "measured" speed in reference model $\omega_{m-r e f}$, activated load torque estimator and correction speed feedback

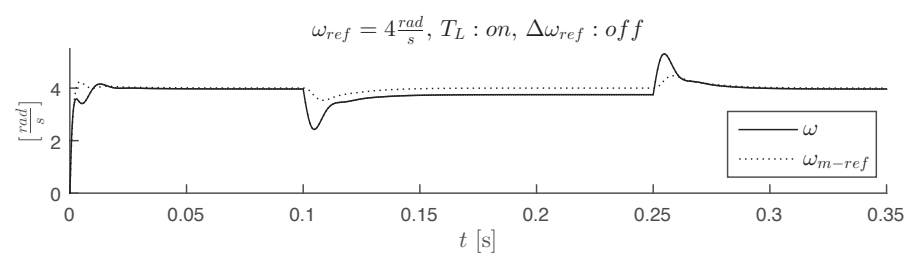

Fig. 7. Simulation, measured speed $\omega$ and "measured" speed in reference model $\omega_{m-r e f}$, activated load torque estimator, deactivated correction speed feedback

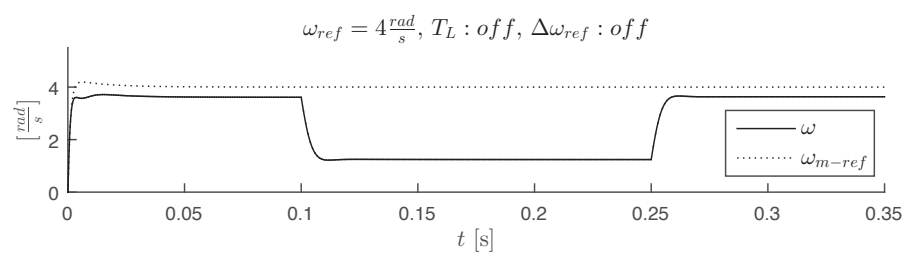

Fig. 8. Simulation, measured speed $\omega$ and "measured" speed in reference model $\omega_{m-r e f}$, deactivated load torque estimator, deactivated correction speed feedback 
is prepared using previously presented equations. The number of pole pairs is equal 1. Resistance of the "real" motor is $50 \%$ greater than the resistance of the motor model, and inductance of the "real" motor is $5 \%$ smaller than the inductance of the motor model. The following simulations were carried out: step change of the reference speed $0 \rightarrow 4 \frac{\mathrm{rad}}{\mathrm{s}}$, then in a time of $0.1 \mathrm{~s}$ the step change of the load to the nominal value, and in a time of $0.25 \mathrm{~s}$ the load was removed. Figure 6 presents the operation in the case where all the correction loops are working. The measured speed achieved the referenced speed in all cases of load conditions. Figure 7 presents the operation in the case where the correction value of the reference speed is not connected. The referenced speed is not achieved in the case of load conditions. Figure 8 presents the operation in the case where all the correction loops are not connected. It is visible that even in such conditions the drive has the possibility to operate. However, the lowest speed for proper operation in all load conditions, on a laboratory stand, is near $4 \frac{\mathrm{rad}}{\mathrm{s}}$. In the case of turning-off only the estimator of the load torque, the drive continues to operate. However, in the case of load condition, the speed oscillates. Figure 9 presents the "measured" current waveforms for testing conditions, as does Fig. 6. It can be concluded that the waveforms in Fig. 6 and Fig. 9 are comparable to experimental waveforms in Fig. 13. Another comparison involves

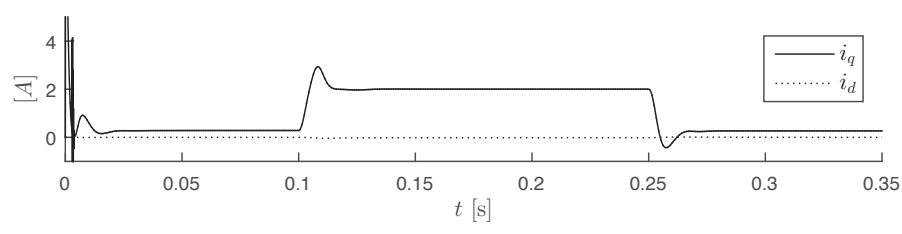

Fig. 9. Simulation, measured current $i_{q}$ and measured current $i_{d}$, under conditions corresponding to these in Fig. 6
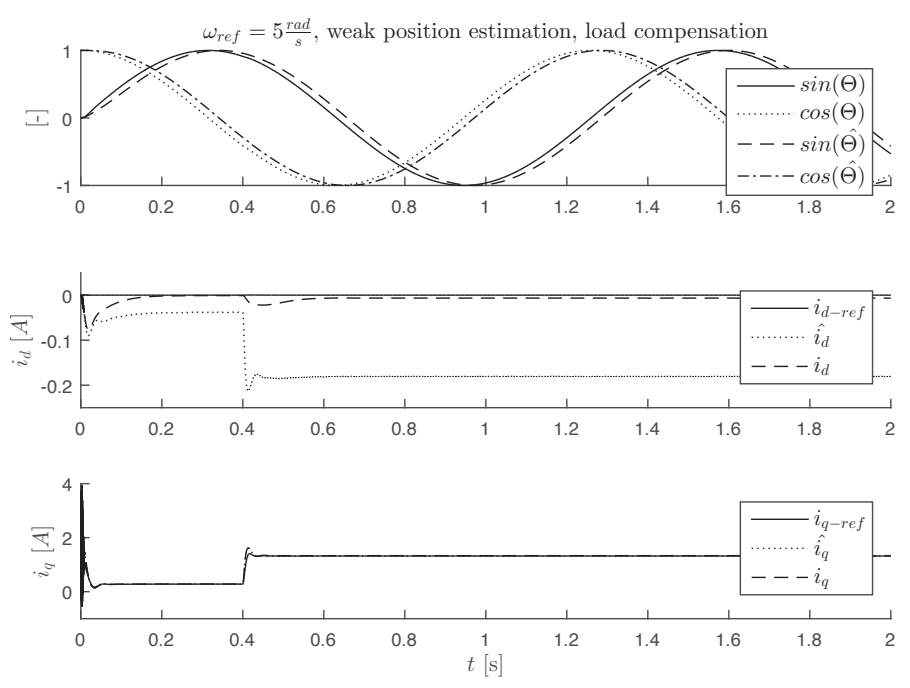

Fig. 10. Simulation, set of waveforms in a case of weak accuracy the position estimation, up: sine and cosine of the shaft position measured and estimated, middle: $d$ axis currents - reference (from model), estimated and measured, down: $q$ axis currents - reference (from model), estimated and measured
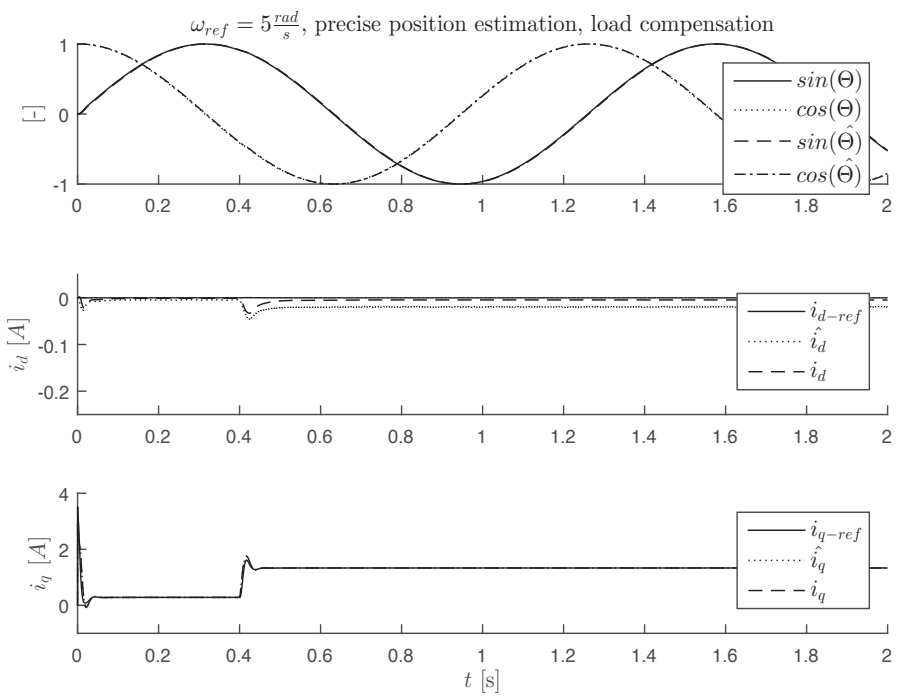

Fig. 11. Simulation, set of waveforms in a case of high accuracy the position estimation, up: sine and cosine of the shaft position - measured and estimated, middle: $d$ axis currents - reference (from model), estimated and measured, down: $q$ axis currents - reference (from model), estimated and measured

the drive performance in the case of different accuracies of the position estimator. The accuracy level is visible as a different value of the time delay between waveforms in Fig. 10(up) and Fig. 11(up). In the first case, position error is about $7.5^{\circ}$, in the second case it is about $0.6^{\circ}$ (the waveforms of sine and cosine are overlapped). At the time $0.4 \mathrm{~s}$, a step change of load torque was introduced. It can be seen that the error value strongly affects the $d$ axis current - Fig. 10(middle), Fig. 11(middle). In the case of weak position estimation accuracy, a stronger correction is necessary (it is visible as a bigger difference between estimated and measured value of $d$ axis current). However, it is noticeable that the position estimation error has a weak influence on $q$ axis current - Fig. 10(down), Fig. 11(down).

\section{Experimental investigations}

The control structure was tested on a laboratory stand involving $1.23 \mathrm{~kW}, 3000 \mathrm{rpm}$ PMSM supplied by a laboratory inverter and a second similar PMSM, working as a controlled load source, supplied by an industrial AC drive. The number of pole pairs is equal 3. The first inverter is fed using a small transformer. It was used to limit the supply voltage at the first stage of the test of the control algorithms. The voltage was reduced to $34 \mathrm{~V}$. The tested drive was controlled by means of a DSP and FPGA based system. The main control system is based on the floating point DSP from SHARC family. The resolver is used only for comparison purposes. The laboratory inverter's carrier frequency was equal to $10 \mathrm{kHz}$. The observer as well as control algorithm were implemented on a DSP processor system. All these programs were executed every $100 \mu \mathrm{s}$. The measurement algorithms were implemented on an addi- 
tional FPGA evaluation board. The main control device was a DSP evaluation board connected to a FPGA board in the form of a sandwich. It was directly connected to the control inputs of a laboratory inverter.

Various tests were carried out to confirm the correct operation of the system. Selected waveforms are shown in Figs. 12 and 13. All waveforms were obtained in sensorless mode, where sine and cosine of the estimated position $\hat{\Theta}$ were used in the control chain. All test results were obtained for low speed range, where speed value was in the range of single revolutions per second. If the motor operates at this operating point, it starts to cover an additional area of the nonlinear phenomena; nonlinearity strongly implicates the drive performance, which is visible even on the sensor mode of operation (Fig. 1), where for a fixed value of the $i_{q}$ current, the resulting speed is not smooth. Obtaining smooth velocity waveforms for this operating point is, however, a separate research task.

Figure 12 presents waveforms in steady state operation, for a system working without load compensation - the system operates directly as is shown in Fig. 3 - and waveforms for an improved structure (Fig. 5), with load compensation. It is visible that the drive works stably in both versions; however, this simple modification significantly reduces ripple of the measured speed and current $i_{d}$ as well as the value of the constant component of current $i_{d}$. In addition, the accidentally recorded disturbances (noise in measured speed waveforms - Fig. 12), affect the speed sensor; however, the position estimation procedure is not affected. The next test confirms the proper operation during a step change of the motor load (Fig. 13) at constant reference speed $\omega_{\text {ref }}=5 \frac{\mathrm{rad}}{\mathrm{s}}$. It can be seen that neither the load switching nor the load value changes the waveform quality. The drive operates stably, regardless of the load. It is confirmed by
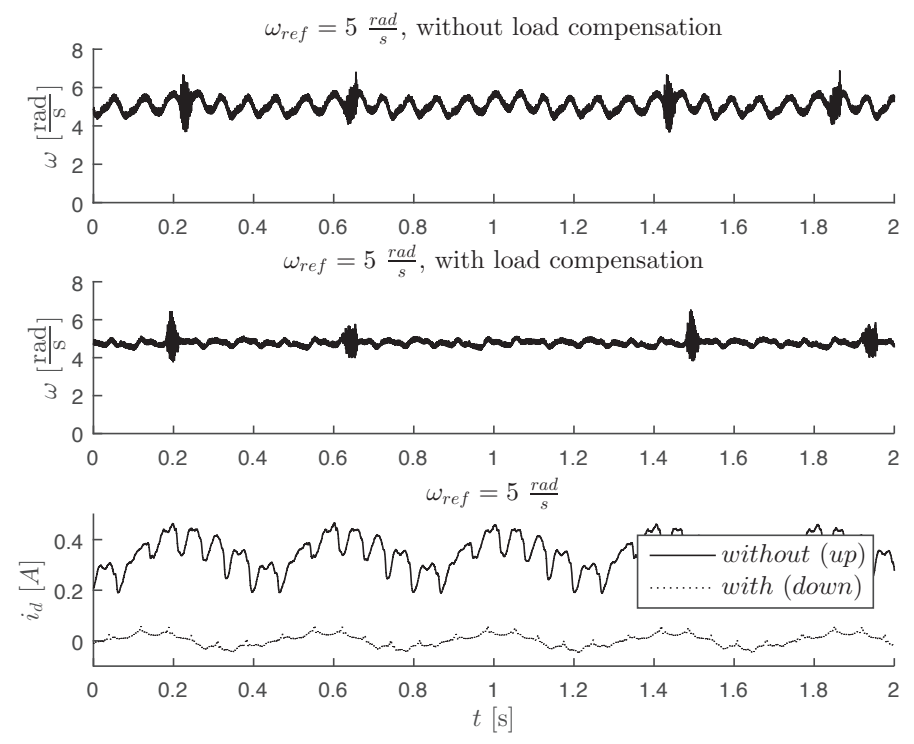

Fig. 12. Experimental investigations, sensorless mode, motor operation with and without load compensation, $\omega_{\text {ref }}=5 \frac{\mathrm{rad}}{\mathrm{s}}$, up: measured speed without load compensation, middle: measured speed with load compensation, down: measured currents $i_{d}$ (up: without load compensation, down: with load compensation)
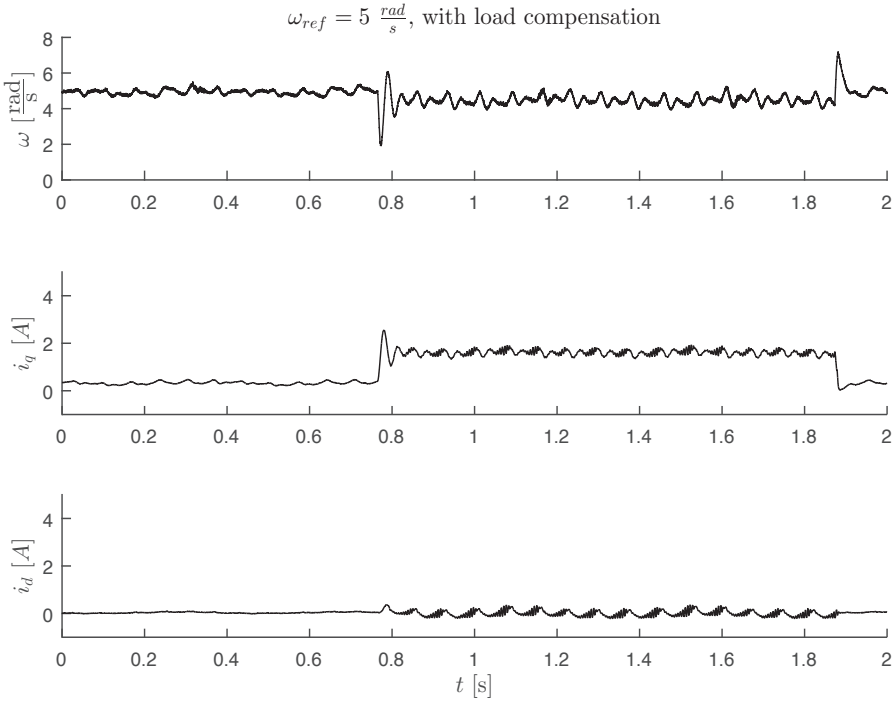

Fig. 13. Experimental investigations, sensorless mode, motor operation with load compensation, rapid changes of load $T_{L}=0 \rightarrow 1.6 \rightarrow 0 \mathrm{~N} \cdot \mathrm{m}$, $\omega_{\text {ref }}=5 \frac{\mathrm{rad}}{\mathrm{s}}$, up: measured speed, middle: measured current $i_{q}$, down: measured currents $i_{d}$

the lack of disturbance in the measured speed waveform and moreover in the low value of current $i_{d}$, whose value depends on the position estimation accuracy. There are no significant changes in the motor speed quality just as there are no excessive oscillations in the current $i_{q}$. A zero reference value of the current $i_{d}$ is maintained at all times with sufficient accuracy, even in dynamic states.

\section{Conclusions}

This control structure was developed to handle the sensorless control, especially in the low speed range. Elimination of the speed calculation, which is based on the estimated back EMF or estimated position, is a big advantage of this control structure. In the case of possible irregularity of estimated back EMF in this speed range, a fast and accurate speed calculation is difficult or impossible. Such a control structure makes it possible to perform a smooth operation of the drive, even in the case of a step change of reference speed. This task is carried out properly with acceptable accuracy and dynamics. Such a control structure works well even for a drive with a small moment of inertia, and works properly even if rapid changes of motor load occur. Using this control method, the motor works silently. Experimental investigations prove the correctness of the concept. A load compensation mechanism allows a significant reduction in the ripple of the speed and current $i_{d}$. The direction of further research will be the decrease of the ripple in the speed or torque, (e.g., using a nonlinear controller), a study of the robustness on inaccurate drive parameter estimation and the robustness on the position estimation error. A complex control system, prepared in Matlab-Simulink, can be easily transferred into the DSP system. 


\section{REFERENCES}

[1] H. Kim, J. Son, and J. Lee, "A high-speed sliding-mode observer for the sensorless speed control of a PMSM", IEEE Transactions on Industrial Electronics 58 (9), 4069-4077 (2011).

[2] L. Li, G. Tan, J. Liu, and B. Kou, "Sensorless control of PMSM based on state observer and the parameter error analysis", in IEEE International Conference on Power Electronics, Drives and Energy Systems, 1-8 (2012).

[3] W. Gao and Z. Guo, "Speed sensorless control of PMSM using model reference adaptive system and RBFN", Journal of Networks 8 (2013).

[4] Y. Fan, L. Zhang, M. Cheng, and K. Chau, "Sensorless SVPWM-FADTC of a new flux-modulated permanent-magnet wheel motor based on a wide-speed sliding mode observer", IEEE Transactions on Industrial Electronics 62, 3143-3151 (2015).

[5] T. Michalski, C. Lopez, A. Garcia, and L. Romeral, "Sensorless control of five phase PMSM based on extended Kalman filter", 42nd Annual Conference of the IEEE Industrial Electronics Society, 2904-2909 (2016).

[6] X. Song, J. Fang, B. Han, and S. Zheng, "Adaptive compensation method for high-speed surface PMSM sensorless drives of EMFbased position estimation error", IEEE Transactions on Power Electronics 31, 1438-1449 (2016).

[7] J. Guzinski and H. Abu-Rub, "Predictive current control implementation in the sensorless induction motor drive", IEEE International Symposium on Industrial Electronics, 691-696 (2011).

[8] K. Urbanski, "Position estimation for PMSM drive equipped with the motor choke", Przeglad Elektrotechniczny 4, 237-241 (2013).

[9] D. Janiszewski, "Load torque estimation for sensorless PMSM drive with output filter fed by PWM converter", in 39th Annual Conference of the IEEE Industrial Electronics Society, 2953-2959 (2013).

[10] J. Salomaki, A. Piippo, M. Hinkkanen, and J. Luomi, "Sensorless vector control of PMSM drives equipped with inverter output filter", 32nd Annual Conference on Industrial Electronics, 1059 -1064 (2006).

[11] S.-M. Gu, F.-Y. He, and H. Zhang, "Study on extend Kalman filter at low speed in sensorless PMSM drives", in International Conference on Electronic Computer Technology, 311-316 (2009).

[12] S. Brock and J. Deskur, "A practical approach to compensation of torque ripple in high-precision permanent magnet motor drives", in International Conference on Electrical Drives and Power Electronics, Dubrovnik (2005).

[13] P. Bogusz, "A switched reluctance motor control method limiting the maximum dc source current in the low-speed range", Bull. Pol. Ac.: Tech. 64(1), 197-206, (2016).

[14] J. Wisniewski and W. Koczara, "The sensorless rotor position identification and low speed operation of the axial flux permanent magnet motor controlled by the novel PIPCRM method", in IEEE Power Electronics Specialists Conference. PESC, 2008.

[15] R. Raute, C. Caruana, C. Spiteri Staines, J. Cilia, N. Teske, M. Sumner, and G. M. Asher, "A review of sensorless control in induction machines using hf injection, test vectors and PWM harmonics", in Symposium on Sensorless Control for Electrical Drives, 47-55 (2011)

[16] M. Schroedl, "Sensorless control of AC machines at low speed and standstill based on the INFORM method", in Conference Record of the 1996 IEEE Industry Applications Conference, 270-277 (1996).
[17] G. Xie, K. Lu, S. Dwivedi, R. Riber, and F. Blaabjerg, "Minimum voltage vector injection method for sensorless control of PMSM for low-speed operations", IEEE Transactions on Power Electronics 31 (2), 1785-1794 (2016).

[18] P. Pelczewski, W. Oberschelp, and U. Kunz, "Optimal model-following control of a positioning drive system with a permanent-magnet synchronous motor", Control Theory and Applications, IEE Proceedings D 138, 267-273 (1991).

[19] X. Dong, W. Tianmiao,W. Hongxing, and L. Jingmeng, “Adaptive model following speed control method of permanent magnet synchronous motor", in 4th IEEE Conference on Industrial Electronics and Applications, 721-725 (2009).

[20] J. Kang, X. Zeng, Y. Wu, and D. Hu, "Study of position sensorless control of PMSM based on MRAS", in IEEE International Conference on Industrial Technology, 1-4 (2009).

[21] R. Mustafa, Z. Ibrahim, and J. Lazi, "Sensorless adaptive speed control for PMSM drives", in Power Engineering and Optimization Conference, 511- 516 (2010).

[22] S. Li and H. Gu, "Fuzzy adaptive internal model control schemes for PMSM speed-regulation system", IEEE Transactions on Industrial Informatics 8, 767-779 (2012).

[23] T. Tarczewski, L. Grzesiak, A. Wawrzak, K. Karwowski, and K. Erwinski, "A state-space approach for control of NPC type 3-level sine wave inverter used in FOC PMSM drive", Bull. Pol. Ac.: Tech. 62(3), 439-448 (2014).

[24] P. Vas, Sensorless Vector and Direct Torque Control, Monographs in Electrical and Electronic Engineering, Oxford- New York, Oxford University Press, 1998.

[25] D. Luenberger, "An introduction to observers", IEEE Transactions on Automatic Control 16, 596-602 (1971).

[26] K. Urbanski and K. Zawirski, "Improved method for position estimation using a two-dimensional scheduling array", Automatika-Journal for Control, Measurement, Electronics, Computing and Communications 56, 331-340 (2015).

[27] F. Benchabane, A. Titaouine, O. Bennis, K. Yahia, D. Taibi, and A. Guettaf, "Sensorless direct torque control for salientpole PMSM based on extended Kalman filter fed by AC/DC/AC converter", Frontiers in Energy 6, 247-254 (2012).

[28] Z. Wang, Y. Zheng, Z. Zou, and M. Cheng, "Position sensorless control of interleaved CSI fed PMSM drive with extended Kalman filter", IEEE Transactions on Magnetics 48, 3688-3691 (2012).

[29] K. Zawirski, D. Janiszewski, and R. Muszynski, "Unscented and extended Kalman filters study for sensorless control of PM synchronous motors with load torque estimation", Bull. Pol. Ac.: Tech. 61 (4), 793-801 (2013).

[30] C. Zych, A. Wronska-Zych, J. Dudczyk, and A. Kawalec, “A correction in feedback loop applied to two-axis gimbal stabilization", Bull. Pol. Ac.: Tech. 63 (1), 217-219 (2015).

[31] Y. Zhao, W. Qiao, and L. Wu, "Compensation algorithms for sliding mode observers in sensorless control of IPMSMs", in Electric Vehicle Conference, 1 -7 (2012).

[32] Z. Qiao, T. Shi, Y. Wang, Y. Yan, C. Xia, and X. He, "New sliding-mode observer for position sensorless control of permanent-magnet synchronous motor", IEEE Transactions on Industrial Electronics 60, 710-719 (2013).

[33] T. Bernardes, V. Foletto Montagner, H. Grundling, and H. Pinheiro, "Discrete-time sliding mode observer for sensorless vector control of permanent magnet synchronous machine", IEEE Transactions on Industrial Electronics 61, 1679-1691 (2014).

[34] A. Accetta, M. Cirrincione, and M. Pucci, "Sensorless control of PMSM by a linear neural network: TLS EXIN neuron", in 36th Annual Conference on IEEE Industrial Electronics Society, $974-978$ (2010). 